\title{
ENGINEERING POTENTIAL FOR LUNAR MISSIONS \\ AFTER APOLLO
}

\author{
JAMES D. BURKE
}

Jet Propulsion Laboratory, Pasadena, California, U.S.A.

\begin{abstract}
With results of past and present lunar exploration programs in hand, it is now possible to outline the next logical steps in the exploration and use of the Moon. This paper describes first, a set of mission objectives and engineering capabilities considered desirable and feasible for projects following soon after Apollo, and second, some objectives for the farther future.
\end{abstract}

\section{Introduction}

Assuming success in the remaining Apollo missions, what are the prospects for continuing lunar achievements? This paper will describe some desirable and feasible missions that could be executed at reasonable cost. There is, of course, no certainty now that the U.S. will carry on such a program, since the required national decisions are still in the future. However, by discussing the options now, we can help to assure that any future program will be efficient and scientifically productive. It is a privilege to present ideas developed at JPL to members of the IAU, for one of our recognized goals is to increase international participation, not only in our present projects but also in the early planning of possible future programs. The IAU, with its long tradition of free and prompt information exchange, can help not only in the scientific review and discussion of our plans, but also in cultivating support for these plans by scientists in other countries, whose efforts may be complementary to our own.

\section{Rationale for Further Lunar Investigations}

At JPL we have proceeded from the viewpoint of an evolutionary cosmogony, regarding the Moon as a source of information on the various stages of a postulated grand process that begins with nucleosynthesis and stellar evolution, continues

\section{TABLE I}

Uses of Moon for investigating evolution

$\begin{array}{ll}\text { Stage of evolution } & \text { Typical lunar investigations } \\ \text { Stellar } & \text { Wide-spectrum astronomy } \\ \text { Planetary } & \text { Selenodesy, geophysics, geology } \\ \text { Biological } & \text { Organic geochemistry, volatiles } \\ \text { Social } & \text { International programs } \\ & \\ \text { Interplanetary } & \text { Communications experiments }\end{array}$


through the formation of planets and life, and extends on into the unknown evolutionary future of life forms and their artifacts, here in the Earth-Moon system or elsewhere. Some of the more obvious uses of the Moon for investigating (or advancing) these stages of evolution are listed in Table I.

\section{A. STELlar EVOLUTiON}

The Moon is already in use as a site for observations relevant to astrophysics. The Apollo solar-wind experiments and the Lunokhod X-ray telescope are examples. However, to reach the goal of founding automated or manned lunar observatories, as long desired by every true amateur of the Moon, astronomers and engineers will have to work together to demonstrate the merits of such observatories in competition with Earth-orbital and Earth-based techniques. Because there are still so many useful observations that can be made from on or near the Earth [1], resources continue to be allocated mainly here, in preference to Moon-based efforts.

To understand this value tradeoff and to predict its future course remain as challenges to those of us who advocate and plan lunar programs; we would be most grateful for advice and assistance from members of the IAU.

\section{B. PLANETARY EVOLUTION}

The idea that the Moon is a source of information dating possibly from the time of planetary accretion has been a main force in past and present lunar exploration programs. During more than a decade at JPL [2] and [3], studies have been made to define lunar investigations pertinent to the problem of the origin and evolution of planets. In our more recent work [4]-[9], we have concentrated on investigations, particularly those involving remotely-controlled lunar surface traverses, that can build upon the results of the Apollo program and so extend our planetological knowledge of the Moon. The engineering prospects emerging from these studies are discussed in Section 3. The scientific requirements include imaging and sampling of lunar surface materials, elemental and mineralogical measurements, and geophysical measurements such as thermal, seismic, and gravity profiles, with the object of determining the source, pre-accretion history, mode of accumulation, and post-accretion history of the lunar material. In addition to their intrinsic interest, these ancient events demand understanding for the broader view: by developing a bulk compositional model and a compatible thermal history for the Moon, we may elucidate the early history of the Earth-Moon system (cf., for example, [10]-[13]) and thence learn more about the conditions required for the origin of life.

Assuming success in all currently-planned Apollo experiments and an adequate data analysis, we can expect to have a thorough understanding of lunar surface properties at six sites, a good passive seismic survey, the beginnings of a good EarthMoon laser ranging network, and a variety of information on geologic processes in the lunar environment. The Apollo orbital experiments, coupled with the results of earlier missions [14] should yield metric photography over broad regions, improved knowledge of the Moon's equatorial geopotential and surface shapes, and a 
variety of remote-sensor data on surface physical and chemical properties from the equator to middle latitudes. Polar surveys and far-side landings are excluded; some engineering aspects of such missions are mentioned in Section 3.

A logical post-Apollo planetological program, then, should include polar lunar orbital surveys, long surface traverses, and continuing Earth-based experiments such as the precision tracking of optical and radio reflectors or beacons on the Moon. There are also applications in planetology for telescopes on the Moon, for example in the spectrometry of planetary atmospheres, but as mentioned earlier the planning of such missions is not now far advanced.

\section{BIOLOGICAL EVOLUTION}

The Apollo lunar surface samples from equatorial maria are anhydrous and practically devoid of organic or proto-organic materials [15]. These regions of the Moon may be representative of one kind of lifeless planetary environment and can therefore serve as controls in future exobiology investigations. In the continuing exploration of the Moon, it will be desirable to search for subsurface and polar volatiles, to determine the composition of highland rocks and soils, and to extend the Apollo results into an understanding of the biological potential of all lunar materials including, for example, carbonaceous meteorite residues. Automated surface traverse missions can be useful in these searches.

Experiments have been made using Apollo samples to test the effectiveness of lunar soil as a growth medium. With reasonable engineering measures such tests could also be made remotely in small environmental shelters on the Moon; however, we are not now pursuing this development.

It remains to be seen whether or not the Moon was a part of a pre-biotic Earth, or whether it had a major role in modifying the Earth's early environment, and so contributed to the local beginning of life. Insight into this problem may be gained through further study of the mechanics of the Earth-Moon system. If so, the precise radio and laser measurements mentioned earlier for planetology may be important also in a biological context.

\section{SOCIAL EVOLUTION}

There can be no doubt that the study of the Moon has played a significant role in the growth and change of human institutions. One has only to recall the development of optical astronomy, celestial mechanics, and navigation to see that this is so. Now, in the act of reaching toward the Moon, we may be increasing our own chance of success as a species [16]. At the very least, the energy and the ingenuity dedicated to lunar programs have been subtracted from the total available for weapons. In a more positive sense, we are now demonstrating the power of large, systematic problemsolving organizations on a scale known previously only in war. And above all, we have opened a new arena for collaboration and stimulating, non-destructive competition [17]. Members of the IAU, because of their long experience in international efforts, are especially well prepared to observe both the prospects and the limitations 
of such programs, and to assist in the practical planning of new lunar ventures to take advantage of this historic opportunity.

\section{E. INTERPLANETARY EVOLUTION}

As the concept of extraterrestrial intelligence passes from the realm of fantasy and speculation into the realm of sober inquiry [18], it is appropriate to consider the role of the Moon in a future stage of evolution characterized by exchanges of information across the cosmos. The most obvious possible use of the Moon is as a site for sensitive radio receivers with large antennas on the far side, shielded from the radio noise of Earth. Without guessing as to the likelihood of having such interplanetary communications at interstellar distances, we can note that, over the long term, the required engineering developments may move forward during radio astronomy research and spacecraft tracking from the Moon.

\section{Engineering Prospects}

\section{A. EARTH-MOON TRANSPORTATION}

One factor governing the design of a post-Apollo lunar exploration program is the available launch-vehicle capacity. For U.S. flights following shutdown of Apollo and Saturn 5 production, the candidate launching systems are (a) Atlas-Centaur, (b) various versions of the Titan 3, and eventually (c) a reusable manned orbital shuttle with added upper stages. Table II lists approximate lunar spacecraft masses [19]

TABLE II

Lunar vehicle performance

\begin{tabular}{lllll} 
& & \multicolumn{3}{l}{ Approximate spacecraft mass, kg } \\
\cline { 3 - 5 } No. & Launch vehicle & $\begin{array}{l}\text { At translunar } \\
\text { injection }\end{array}$ & $\begin{array}{l}\text { In lunar } \\
\text { orbit }\end{array}$ & $\begin{array}{l}\text { On lunar } \\
\text { surface }\end{array}$ \\
\hline I & Atlas-Centaur & 1500 & 1000 & 500 \\
II & Titan 3 C & 3000 & $\begin{array}{l}(200)^{\mathrm{a}} \\
(50)^{\mathrm{a}}\end{array}$ & $\begin{array}{l}1000 \\
(150)^{\mathrm{a}}\end{array}$ \\
III & $\begin{array}{l}\text { Shuttle/Up. Stg. } \\
\text { (or Titan 3/ }\end{array}$ & 6000 & $\begin{array}{l}(500)^{\mathrm{a}} \\
(000\end{array}$ & $\begin{array}{l}2000 \\
(300)^{\mathrm{a}}\end{array}$ \\
\hline
\end{tabular}

a Instrument payload portion

and scientific payloads for representative members of this launch vehicle family. Figures 1 and 2 show present versions of the Atlas-Centaur and Titan 3, and Figures 3 and 4 show the approximate spacecraft size envelopes available within the AtlasCentaur (OAO) and Titan-Centaur (Viking) nose fairings. Figure 5 shows the size of these vehicles relative to Saturn 5 . 


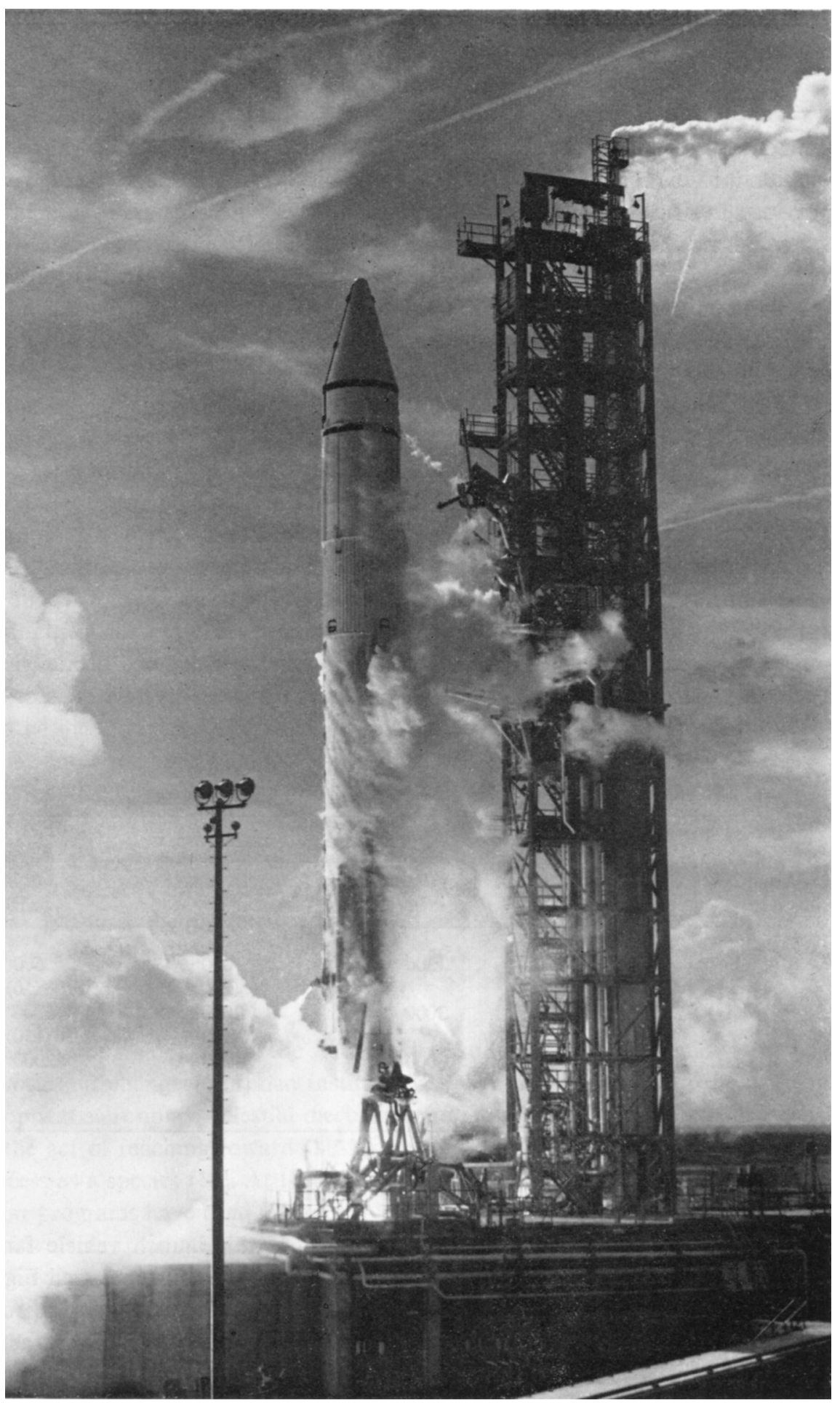

Fig. 1. Atlas-Centaur at Liftoff. 


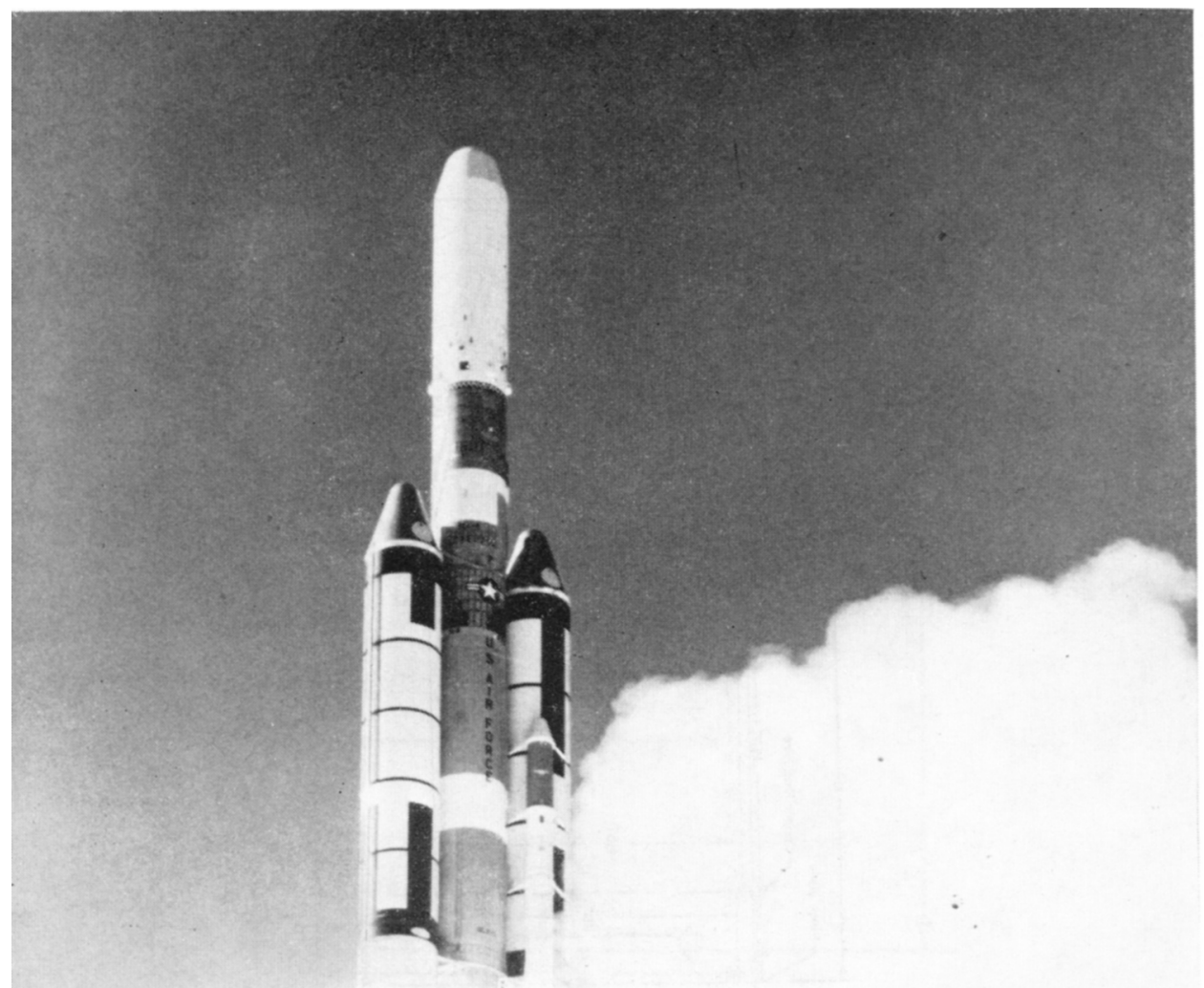

Fig. 2. Titan 3 vehicle shortly after launch. 


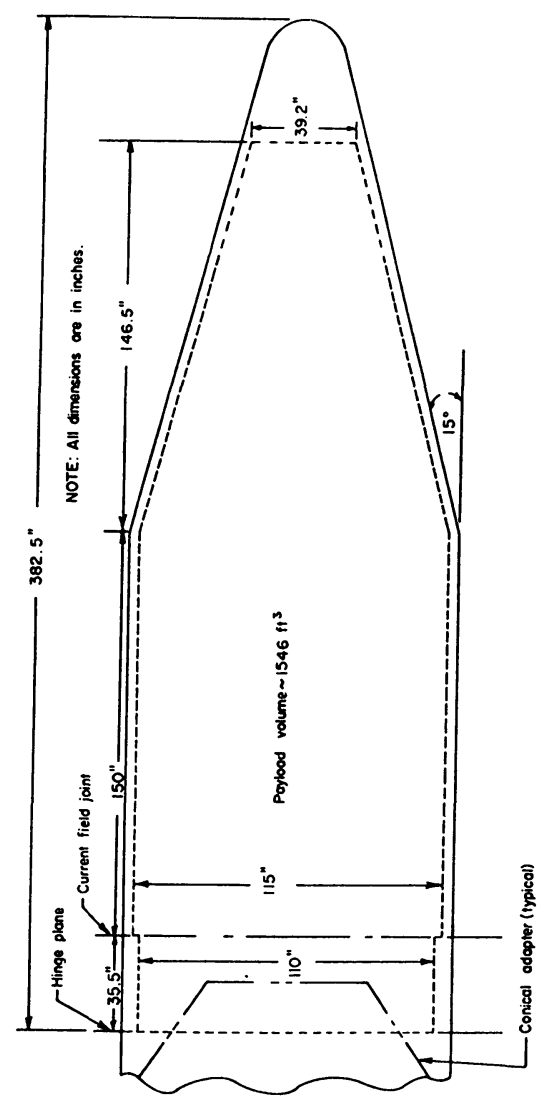

Fig. 3. Centaur nose fairing dimensions for Orbiting Astronomical Observatory.

\section{B. SPACE VEHICLE AND PAYLOAD COST}

Another factor governing program design is, of course, cost. With limited budgets, it may not be possible to use the most powerful available versions of the Atlas and Titan-based launch vehicles, simply because of the higher cost of the larger spacecraft and scientific payloads. For this reason our studies include missions of relatively small capability in comparison to Apollo-missions which may not require the greatest lifting capacity available at a given future date. Even with this restriction, program costs may amount to hundreds of millions of dollars over the several years when a project is active. However, our studies and experiments show that very important data can be obtained with these modest payloads.

\section{LUNAR ORBITAL MISSIONS}

A class of experiments important in planetology can be carried out in lunar orbit [14]. The planned Apollo CSM instrumentation will be very useful, but the Apollo mission constraints prohibit achievement of highly-inclined orbits. Therefore one candidate post-Apollo mission is a polar orbiter with instruments similar to or developed from 

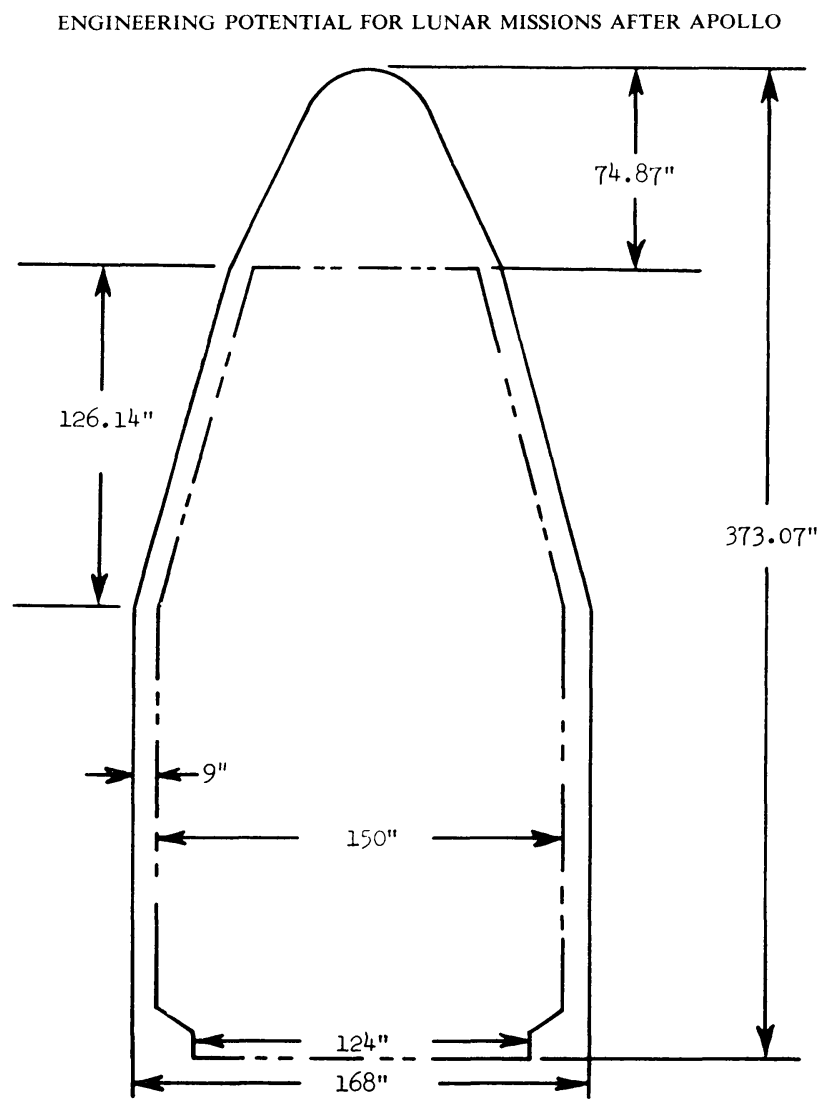

Fig. 4. Centaur nose fairing dimensions for Viking.

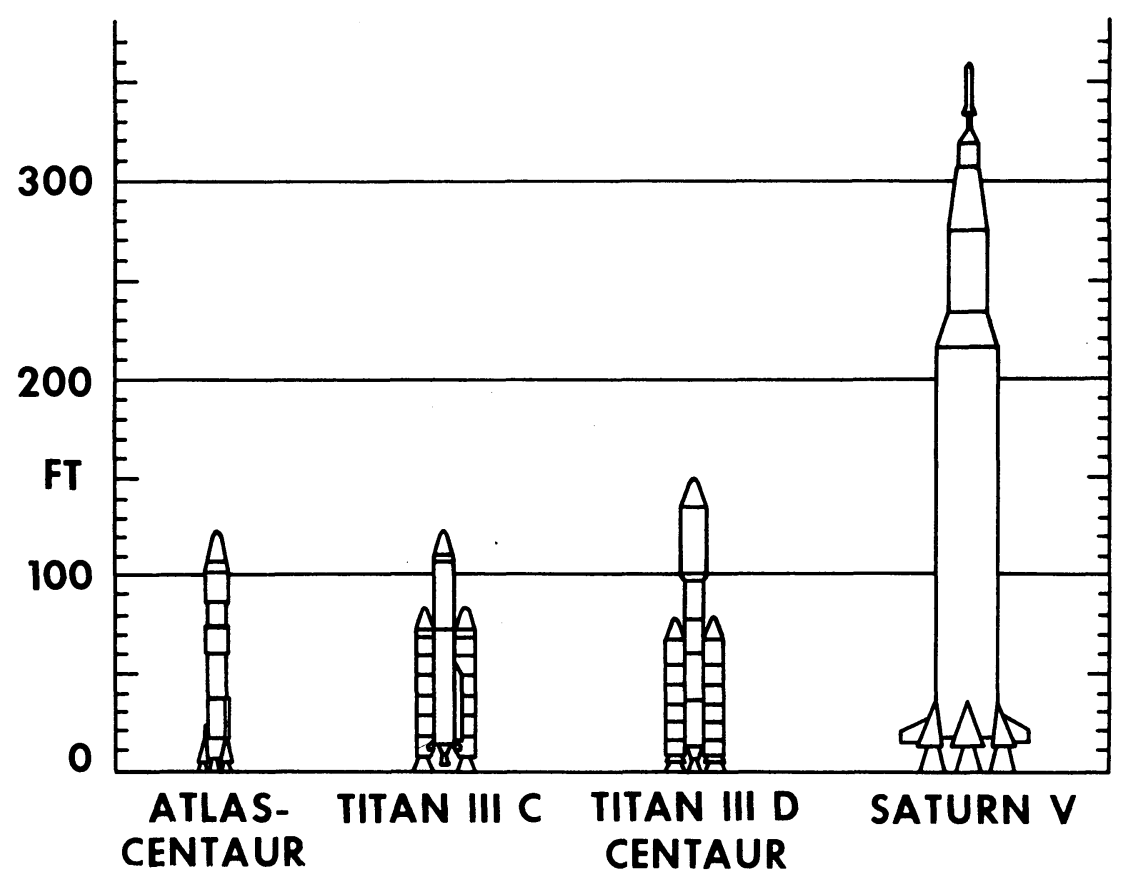

Fig. 5. Size comparison of launch vehicles. 
those to be flown in the later Apollo CSM spacecraft. Even a simple polar orbiter, with just a radar altimeter, a gravity gradiometer, and a precision tracking transponder, could yield important planetological information.

The discussion of post-Apollo lunar orbital missions is complicated by the large number of practicable options. One can visualize useful results from a variety of missions extending from small, simple particle-and-field surveys to elaborate multipurpose missions requiring complex and expensive spacecraft. A logical program would include at least those measurements necessary to define the meridional geopotential and surface figures of the Moon, plus simple compositional remote-sensing measurements such as a gamma-ray survey. If a multi-frequency radio sounder could be carried, data might be obtained on subsurface temperatures and the presence or absence of permafrost in polar regions. Should a more elaborate effort prove possible, one or more metric and remote-sensing experiments based on any of several radiations and detector principles could be added. The main purpose of such measurements would be to extend, by a combination of observation and inference, verified point data from surface missions to larger areas.

\section{LUNAR SURFACE TRANSPORTATION}

Mobility on the lunar surface is essential for the conduct of most of the important

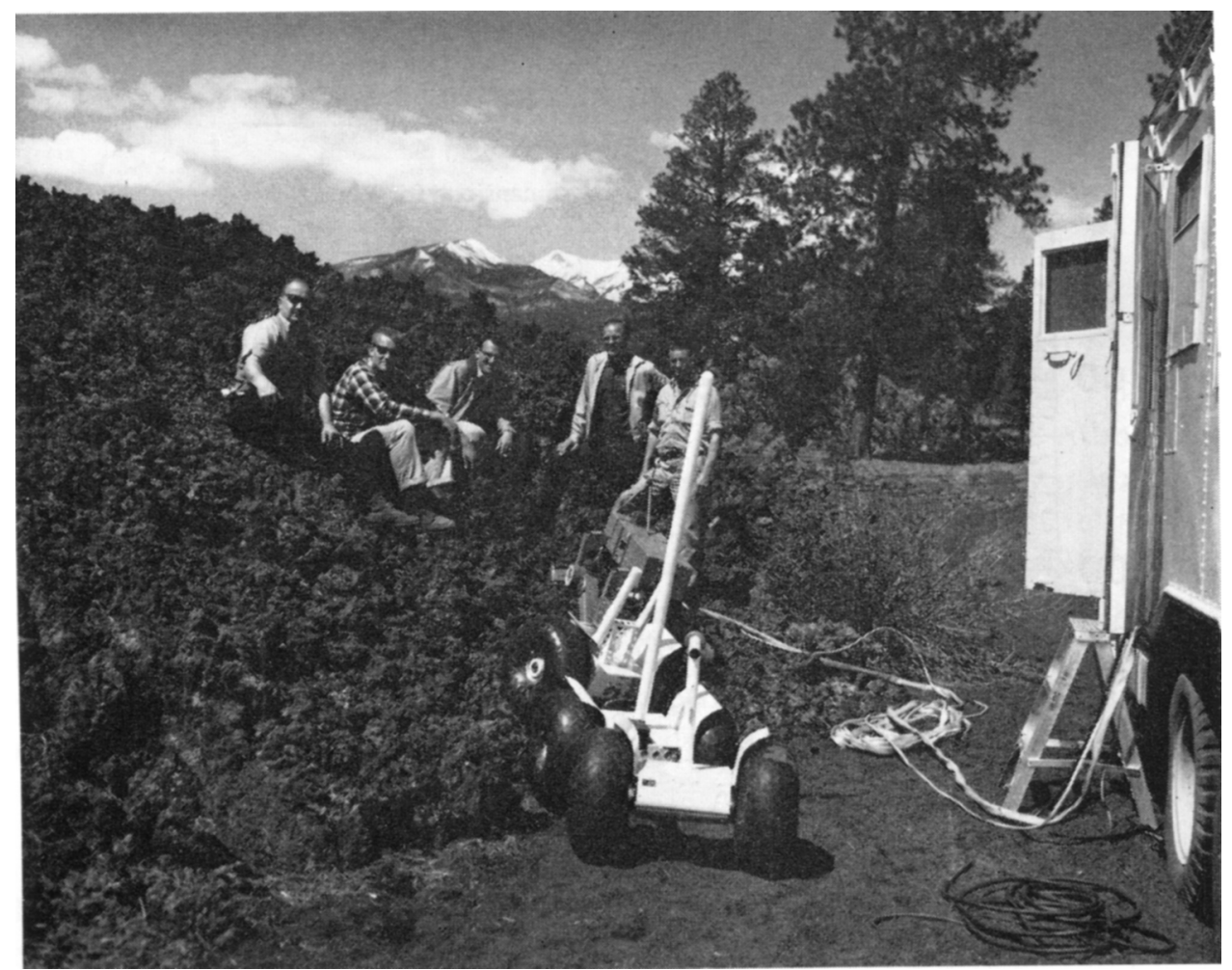

Fig. 6. Surveyor lunar roving vehicles during field test, 1964. 
post-Apollo investigations. The technical foundation for such missions has been laid by developments such as the Surveyor Lunar Roving Vehicles (Figure 6), the Lunokhod, and the Apollo manned lunar rover. Post-Apollo scientific criteria require a long range (hundreds of $\mathrm{km}$ ), and this raises several interrelated engineering problems which we have analyzed. The more important tradeoffs are summarized in Table III.

TABLE III

Engineering tradeoffs for automated long traverses

- Mobility and Hazard Avoidance

- Power, Speed, Range and Endurance

- Image Data Rate, Antenna Gain and Pointing Control

- Guidance and Navigation

- Automation and Human Intervention

\section{Mobility and hazard avoidance}

A basic requirement is that the machine must safely negotiate most of the surfaces encountered, and must avoid, with a certain margin of safety, surfaces on which it could overturn or get stuck. Obviously there is a tradeoff between high mobility, with attendant weight, power and complexity of chassis, wheels and drives, and high hazard-avoidance ability, with complex detection and reaction schemes. Our studies and tests, supported by those of General Motors and several other U.S. organizations, show that for automated missions high mobility (and consequently a more limited demand for hazard avoidance) is the preferred choice. Very good mobility (with acceptable weight and power) can be obtained through the use of a multiwheel, articulated design with low footprint pressure. A similar choice was made by the Lunokhod designers. The manned Apollo rover, on the other hand, can and does have lower basic mobility since its operator is able to judge directly and avoid hazards.

\section{Power, speed, range and endurance}

Power supply is one of the main problems of the automated traverse. For a machine large enough to carry the desired payload (including at least sampling, imaging, and rudimentary sample analysis), the average power for travel and picture transmission may be several hundred watts, and the peak or emergency demand may exceed a kilowatt. There is a complicated tradeoff among speed, stop-go duty cycle, picture data rate, and other variables. In our studies this has been integrated with groundcontrol and hazard-avoidance considerations, to yield the conclusion that average speeds on a long traverse will be only one to a few $\mathrm{km} \mathrm{hr}^{-1}$ - equivalent to a slow walk - and that therefore if it is to travel for hundreds of kilometers the machine must repeatedly survive the lunar night. Lunokhod 1 , though it has not traveled so far, has now demonstrated the important night-survival function. The basic problems of equipment lifetime in the lunar environment now appear to be understood, as a 
result of Surveyor, Apollo, and Soviet experience. Analyses of Surveyor 3 parts returned by Apollo 12 [20] and [21] and ALSEP operating experience lend confidence to this conclusion. Since the roving mission must extend over several months, the prime power source must be either solar or nuclear, with a rechargeable battery to handle demand peaks.

\section{Image data rate, antenna gain and pointing control}

Another main problem is the delivery of pictures to the control site, with sufficient quality and frequency to permit human intervention when needed, either as a supplement to vehicle-borne hazard sensors or as an aid to steering, navigation, and scientific mission planning. This tradeoff works out in an interesting way. Tests by the Bendix Corporation have shown that there is an optimum picture frequency. With cameras mounted rigidly to the vehicle (i.e., not servo-stabilized), operator performance is actually better when still pictures are presented, at intervals of one to a few seconds, than it is when imaging is more nearly continuous, as in a cine film at tens of frames per sec. This helps, of course, with the power problem, since the rapid sending of television-quality images from the Moon requires a large radio power-gain product. Given a practical S-band transmitter of, say, $20 \mathrm{~W}$ output, and assuming reception by the 26-m antennas of the Deep Space Net or the Manned Space Flight Net, the imaging tradeoffs then require a vehicle antenna of modest gain, with a beamwidth of at least several degrees. Higher gain would reduce the power demand but would require a more complex system to keep the beam pointed at Earth despite vehicle motions. Therefore our lunar rover conceptual designs include S-band antennas with apertures of $30 \mathrm{~cm}$ or so, rather small in comparison, for example, to a Mariner antenna.

\section{Guidance and navigation}

Navigation studies and tests [24] show that the only practical scheme for small, longrange automated lunar rovers is visual landmark navigation, supplemented by simple celestial references such as Sun and Earth sensors, and by radio or laser ranging. Inertial systems, including the necessary stellar references, are too complex for this application.

\section{Automation and human intervention}

In all of these design tradeoffs, there is of course the question of how much the machine should know how to do, and how much its masters on Earth must do. With the lunar round-trip delay of a few seconds, plus the picture interval and human reaction times, it is clear that at a minimum the rover must be able to sense a hazard and stop before entering it. If it cannot do so, then it must travel slowly enough that the operator can intervene and stop or redirect its motion, based on his present (slightly obsolete) data. These relationships set limits on, for instance, the resolution of the TV system, the camera fields of view, the nominal cruising speed, the tolerable Sun angles, and the stop-command thresholds of devices such as tilt sensors. On 
the Moon, reasonable speed and safety can be achieved with quite limited autonomy, as demonstrated by the Lunokhod system. For roving on Mars, a much higher degree of autonomy would be required because of the greater communications time delay and the more limited data bandwidth.

\section{E. INSTRUMENTATION}

As pointed out in Section 2, the primary functions visualized for early lunar longtraverse instrument payloads are planetological: geologic reconnaissance, sampling and analysis of surface materials, and geophysical profiling. These experiments would be part of an integrated investigation consisting of (a) the existing diverse and detailed local observations from Apollo sites, (b) simpler observations over regional distances along the traverses, and (c) still less detailed, but broad and synoptic, observations from lunar orbit. Emphasis in the investigation as a whole would be placed on information useful in constructing bulk compositional models for the Moon, in deciphering the Moon's thermal or energy history, and in describing its early interactions with the Earth.

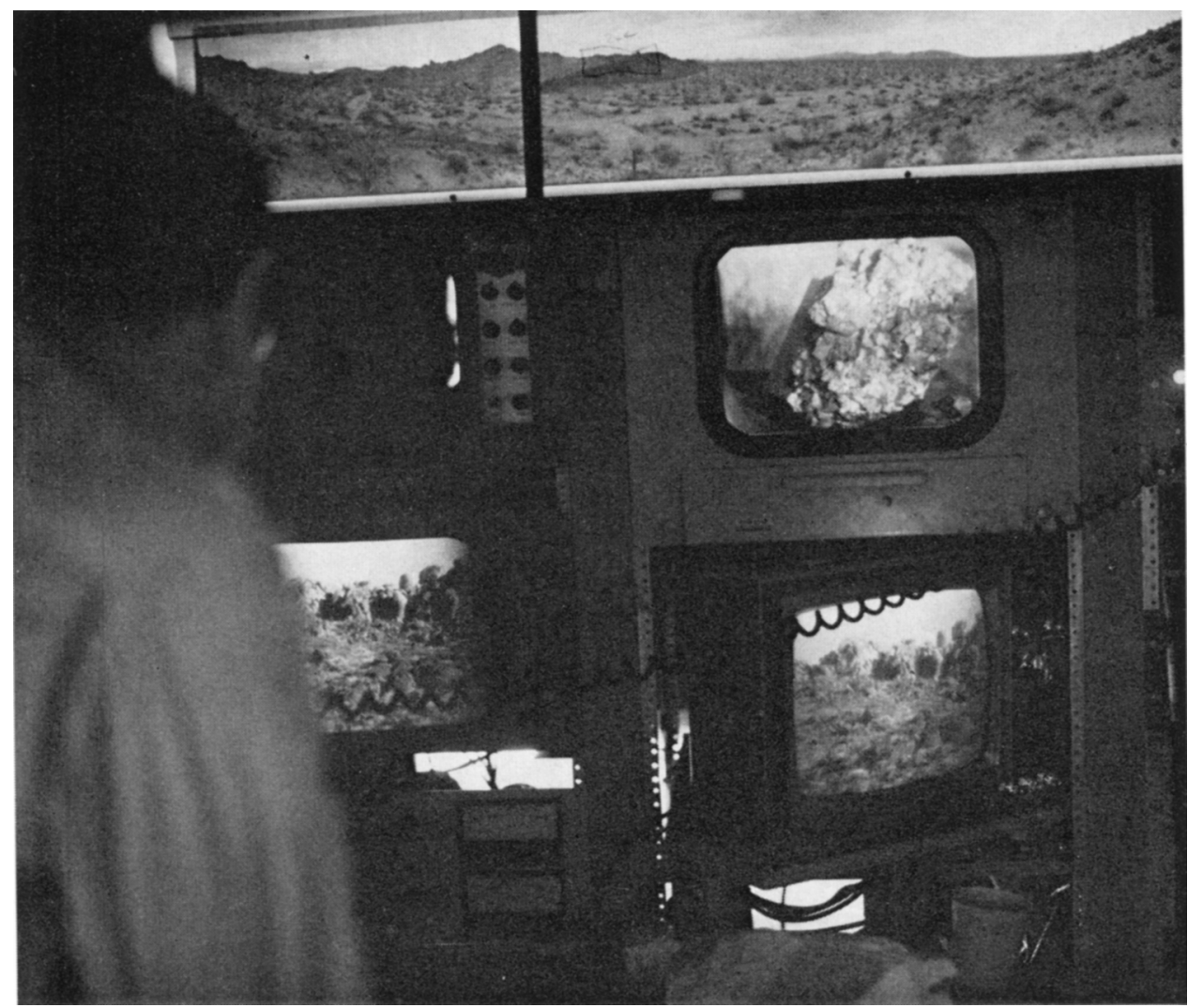

Fig. 7. Visual displays inside field-test truck. Top: panorama, simulating photofacsimile display. Center: TV close-up of rock sample. Bottom: two views of local scene. 


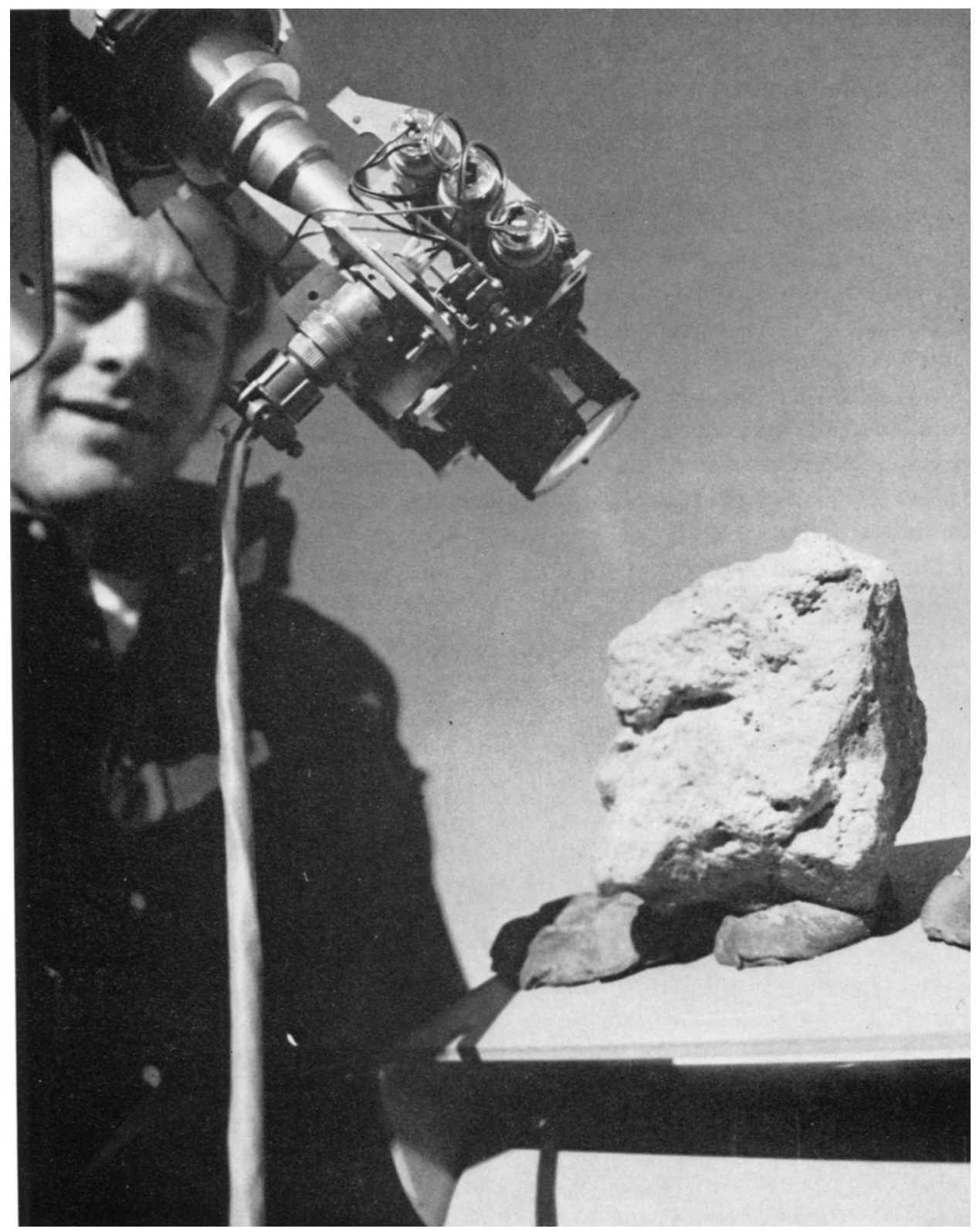

Fig. 8. Rock sample viewed by TV camera. Sample and camera orientation, and zoom lens focus and magnification, are remotely controlled. 
For these purposes the rover must have at least an imaging system designed for both general surveys of surroundings and close-up petrologic examination of samples, plus limited instrumentation (e.g., a scanning electron microprobe) for measuring the chemical content of lunar materials. Desirable additions would be instruments (e.g., an X-ray diffractometer/spectrometer) for discriminating among minerals, and instruments such as radio sounders, magnetometers, gravimeters, or seismometers for examining subsurface properties. A heat-flow experiment would be very desirable, but would be difficult on an automated rover, because of the precise down-hole temperature-gradient measurements required by present techniques.

In view of these desiderata, the JPL study team decided to concentrate on imaging and sampling as the most basic requirements, and we have made various experiments [7], [22] and [23] in this connection. Figures 7 and 8 show a recent field exercise on volcanic terrain in the California desert. A vehicle is set up with cameras and TV monitors viewing the local scene. The navigator and geologist are brought to the site blindfolded and placed inside, with maps and overhead photos simulating what would be available from lunar orbiters. Then, using only what they can see via television, they direct a sampling and mapping operation. The object is to determine various system design parameters, such as how much time is needed for the requisite

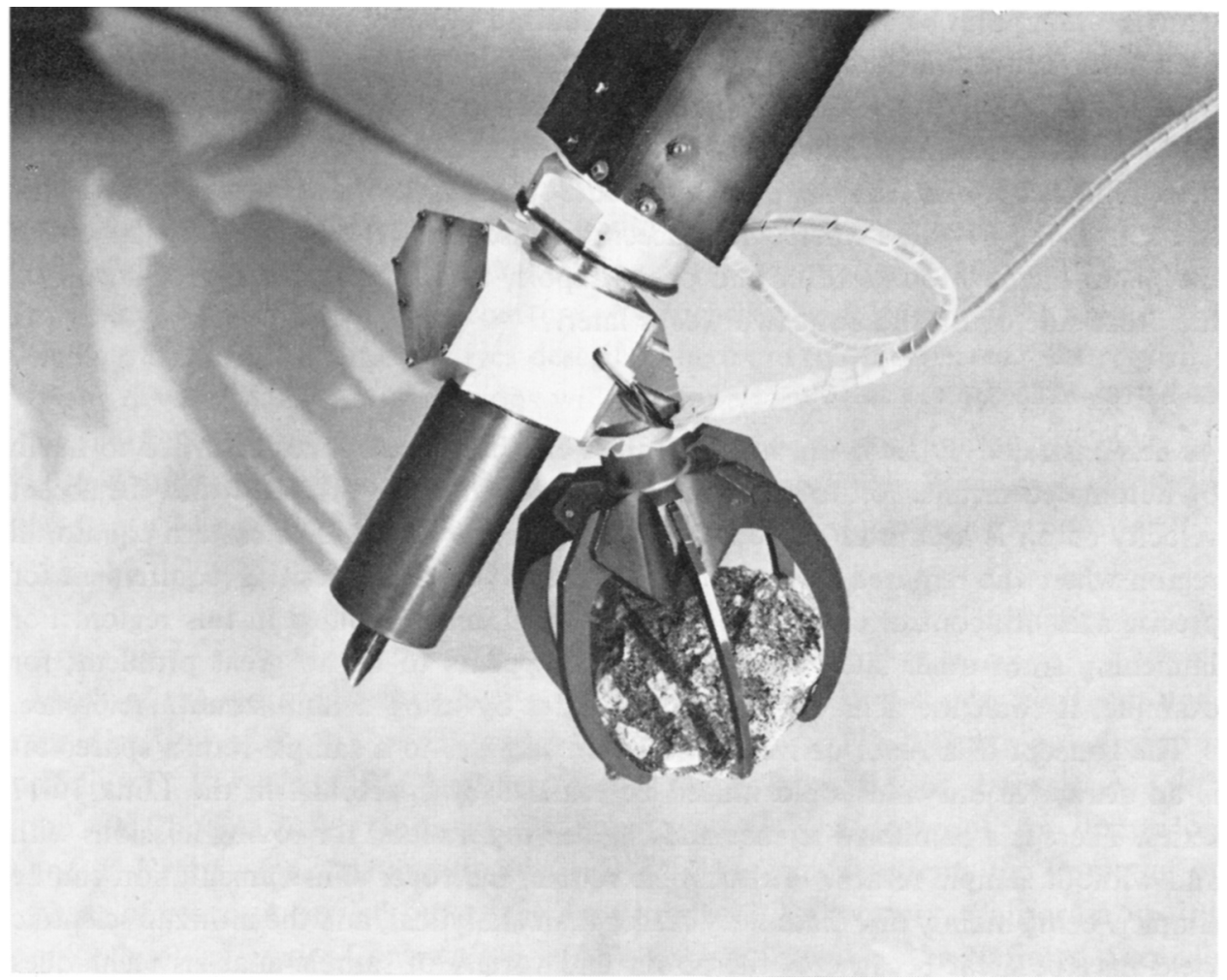

Fig. 9. Chipper-tong device for collecting and manipulating rock samples. 
judgments, how good must the images be, which types of displays are preferred, and so forth. One result, for instance, has been the finding that mineral identification is quite good with a sample-viewing ability as shown in Figure 8, provided that the sample and camera can be manipulated to provide various magnifications and viewing and illumination angles. Samples must therefore be held so that they can be viewed, not enclosed as by the Surveyor scoop. Figure 9 shows a chipper-tong combination designed with this in mind. On the basis of these and other similar tests, we agree with our Soviet colleagues [24] that a lunar rover should have two types of cameras: a photofacsimile device - they call it a telephotometer - for panoramic, high-precision viewing, and a television camera or cameras for driving, where speed of display is more important than picture quality. Color imagery is not important in viewing the general lunar scene, but it is very desirable for the close-up examination of samples.

\section{F. POLAR AND FAR SIDE MISSIONS}

As mentioned earlier, Apollo exploration is limited to middle-latitude orbits and to surface sites on the Earthward side of the Moon. Ultimately we will want access to the surface in polar and far-side regions that do not have line-of-sight to Earth. To support such missions a communications relay will be needed. A promising concept is the 'halo orbit' [25] in which a relay satellite can be located near the Lagrangian libration point beyond the Moon and stabilized by small station-keeping impulses on a path that is continuously visible from Earth.

Landings in polar or far-side regions can be achieved via lunar orbit, as in Apollo and current Soviet automated missions. However, direct Earth-Moon transfers to these regions are also feasible, using slow outbound trajectories. There is even a class of lunar swing-by paths where the spacecraft passes near the Moon, then up out of the plane of the Moon's orbit and over its pole, to encounter the Moon again on the other side of Earth about two weeks later.

\section{G. AUTOMATED SAMPLE RETURN}

As demonstrated by Luna 16, lunar samples can be collected and returned to Earth by automated means. Return-path guidance is not needed, provided that the ascent velocity cutoff is accurate. There is a locus of sites in the Moon's eastern equatorial region where the required ascent vector is vertical; this removes the requirement for precise azimuth control of the launch direction. Luna 16 landed in this region. For launching from other sites, azimuth control appears to be no great problem; for example, it could be achieved at high latitudes by using a Sun-azimuth reference.

The concept of a rover delivering collected samples to a sample-return spacecraft is an attractive one and could indeed be realized by spacecraft in the Luna 16-17 series. There is a combined science and engineering tradeoff for roving missions with and without sample return. With sample return, the rover's instrumentation can be simpler, being mainly discriminatory rather than analytical, and the ultimate scientific yield may be great because of the power and variety of sample-analysis techniques available on Earth. However, for this value to be realized, the rover must make it 
all the way to the rendezvous (or must itself carry the sample-return rocket). Therefore the mission risk is increased. Also, the more limited the rover's instrumentation, the more restricted is its repertoire of actions in response to new findings along the traverse. Since automated missions are fundamentally less versatile than manned ones in this regard, this may prove to be a significant constraint. We therefore conclude that even if sample return is provided, it will be desirable to maximize the rover's analytical ability; this has the added advantage that less would be lost in the event of a failure somewhere along the way.

If the continued delivery of selected samples is assumed, the program plan tends to emphasize instrumentation for sample selection. If not, there must be increased emphasis on sample analysis by remote methods on the Moon. Clearly the latter method cannot provide the variety and precision of Earth-based analytical methods, so that more is dependent on inference. In either case, the suite of samples already gathered provides an essential point of departure. Looking ahead, it is probably desirable to develop at least some of the feasible remote-analysis techniques to prepare for exploring the surface of Mars, whence the return of samples is much more difficult than from the Moon.

\section{Summary and Conclusion}

We have seen that there are numerous opportunities for useful post-Apollo lunar activities. It is to be emphasized that our approach presumes the successful completion of the Apollo program. The kinds of remotely-controlled investigations that appear practical from an engineering and programmatic standpoint are not competitive with manned missions; rather, they should be designed to supplement and extend the results of present programs, making use of the Apollo and Soviet sample analyses and other data as a point of departure. As scientific knowledge and opinion evolve, changes in the program objectives described here are to be expected. But regardless of the detailed plan, lunar missions will continue to offer a fruitful opportunity for international efforts, a new means for observing the cosmos, and an understanding of the boundary conditions for the beginnings of life on Earth. Progress in all of these fields can be advanced by a suitable set of scientific missions to the Moon.

\section{Acknowledgements}

Much of the work described here was done by the JPL Lunar Studies Team under the direction of R. B. Coryell, and by members of the JPL Planetology Group including D. B. Nash, J. E. Conel, and F. P. Fanale. I thank L. D. Jaffe, R. A. Lyttleton, R. Choate, N. H. Horowitz, H. Bank, and G. K. Hornbrook for discussions, and T. Krupka for assistance in preparation. This paper presents the results of one phase of research carried out at the Jet Propulsion Laboratory, California Institute of Technology, under Contract No. NAS7-100, sponsored by the National Aeronautics and Space Administration. 


\section{References}

[1] 'A Long-Range Program in Space Astronomy', Position Paper of the Astronomy Missions Board, NASA SP-213, Washing ton: July 1969.

[2] Hibbs, A. R.: 1959, 'Exploration of the Moon, the Planets, and Interplanetary Space', studies conducted for the National Aeronautics and Space Administration under Contract No. NASw-6, JPL TR 30-1, April 30.

[3] Adams, J. B., Conel, J. E., Dunne, J. A., Fanale, F. P., Holstrom, G. B., and Loomis, A. A.: 1969, Rev. Geophys. 7, No. 3.

[4] Nash, D. B.: 1967, 'Sampling of Planetary Surface Solids for Unmanned in situ Geological and Biological Analysis: Strategy, Principles, and Instrument Requirements', TR 32-1225, Jet Propulsion Laboratory.

[5] Brereton, R. G.: 1968, 'Science Utility of Automated Roving Vehicles', SPS 37-51, Vol. III, Jet Propulsion Laboratory.

[6] Brereton, R. G.: 1968, 'The Objectives for Roving Vehicles in a Lunar Exploration Program', SPS 37-52, Vol. III, Jet Propulsion Laboratory.

[7] Brereton, R. G.: 1969, 'Comments on Geological Observations from an Automated Vehicle (Field Test)', SPS 37-55, Vol. III, Jet Propulsion Laboratory.

[8] Brereton, R. G.: 1969, 'Lunar Surface Gravity Investigations', SPS 37-57, Vol. III, Jet Propulsion Laboratory.

[9] Brereton, R. G.: 1969, 'Imaging and Sampling Requirements for an Automated Roving Vehicle', SPS 37-60, Vol. III, Jet Propulsion Laboratory.

[10] Cameron, A. G. W.: 1970, Trans. AGU 51, 628.

[11] O'Keefe, J. A.: 1970, Trans. AGU 51, 633.

[12] Singer, S. F.: 1970, Trans. AGU 51, 637.

[13] Urey, H. C.: 1971, Science 172, 403.

[14] Allenby, R. J.: 1970, Space Sci. Rev. 11, 5.

[15] 'Apollo 12, Preliminary Science Report', Prepared by NASA Manned Spacecraft Center, NASA SP-235, 1970.

[16] Symington, J. W.: 1970, in " "For the Benefit of All Mankind." A Survey of the Practical Returns from Space Investment', Report of the Committee on Science and Astronautics, U.S. House of Representatives.

[17] 'Space Cooperation Between the United States and the Soviet Union', Hearing before the Committee on Aeronautical and Space Sciences, U.S. Senate, March 17, 1971.

[18] Extraterrestrial Life: An Anthology and Bibliography, compiled by Shneour, E. A., and Ottesen, E. A., Publication 1296A, National Academy of Sciences, National Research Council, Washington, D.C., 1966.

[19] 'Launch Vehicle Estimating Factors', for use in Advance Space Mission Planning, NHB 7100.5, NASA, U.S. Government Printing Office, January 1971.

[20] 'Test and Evaluation of the Surveyor III Television Camera Returned from the Moon by Apollo XII', Hughes Aircraft Company, December 31, 1970.

[21] Jaffe, L. D.: 1970, Science 170, 1092.

[22] Strand, J. N.: 1970, 'Tests of Instruments Proposed for the Scientific Payload of a Lunar Rover', SPS 37-62, Vol. III, Jet Propulsion Laboratory, March 24, 1970.

[23] Burke, J. D., Choate, R., and Coryell, R. B.: 1971, JPL. Quart. Tech. Rev. 1, 131.

[24] Brereton, R. G., Burke, J. D., Coryell, R. B., and Jaffe, L. D.: 1971, JPL. Quart. Tech. Rev. 1, 125.

[25] 'The Utilization of Halo Orbits in Advanced Lunar Operations', Farquhar, R. W., GSFC Document No. X-551-70-449, Goddard Space Flight Center, December 1970. 